\title{
SwissDRG: les médecins resteront les garants de la qualité
}

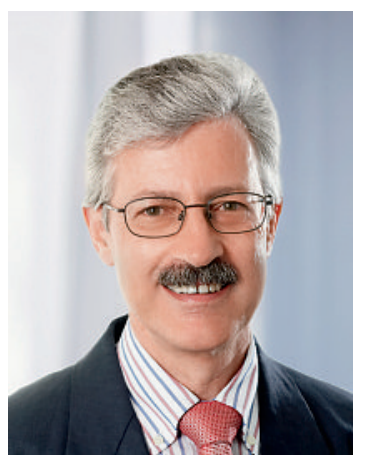

P.-F. Cuénoud
Les préparatifs de la nouvelle structure tarifaire hospitalière SwissDRG vont bon train. Même si son introduction n'est prévue que dans un peu plus de deux ans, il est nécessaire d'implémenter le plus rapidement possible les modifications propres à l'utilisation dans notre pays. A ce point de vue, les partenaires actionnaires de SwissDRG SA ont pris leurs responsabilités et font preuve de bonne volonté dans un esprit constructif, sortant des dialectiques de blocage habituelles. La constitution d'une Société Anonyme semble ainsi avoir été une démarche positive, chacun se sentant concerné et intéressé à ce que l'entreprise réussisse.

Au niveau pratique, le Casemix office a pris ses activités et, avec des moyens limités, abat un travail considérable. En revanche, certaines instances également directement concernées, mais ne dépendant pas des partenaires de SwissDRG SA, ne semblent pas avoir réalisé les enjeux du projet ni le calendrier que cela implique: en particulier, l'Office Fédéral de la Statistique n'a pas reçu les moyens nécessaires à une intégration correcte des propositions de nos Sociétés de disciplines dans la nouvelle édition du code des procédures CHOP-12. $S$ 'il est clair que la première version appliquée de SwissDRG ne pourra pas couvrir de manière fiable la totalité des séjours hospitaliers, un taux d'au moins $80 \%$ devrait être atteint. Pour y parvenir, une sérieuse montée en puissance de ces organismes actuellement insuffisants est de mise.

\section{Il est d'autant plus important de pouvoir objectiver les effets réels du passage à SwissDRG, avec défini- tion de la causalité des phénomènes appréhendés}

Notre pays a la particularité de connaître, dans certains de ses cantons, une rétribution par des forfaits de type DRG depuis plusieurs années. L'introduction de SwissDRG au
1.1.2012 représente donc un changement de système à signification variable suivant les régions. Il est d'autant plus important de pouvoir objectiver les effets réels du passage à SwissDRG, avec définition de la causalité des phénomènes appréhendés: c'est le sujet de la recherche concomitante, à laquelle ce numéro du BMS est en grande partie consacré.

\section{Si la qualité structurelle et la qualité des processus peuvent être objectivées en temps réel, la qualité des résultats nécessite une observation prolongée systématique, sur des bases reconnues}

Toute une série d'indicateurs ont été identifiés, mesurables immédiatement ou à moyen terme. En revanche, la détermination à long terme de la qualité de notre travail ne fait pas partie de cette recherche concomitante et restera, comme auparavant, du ressort des prestataires. En effet, les médecins sont les seuls à connaître l'évolution de leurs patients au fil du temps. Si la qualité structurelle et la qualité des processus peuvent être objectivées en temps réel, la qualité des résultats nécessite une observation prolongée systématique, sur des bases reconnues. Ainsi, nos travaux scientifiques, tels que publiés régulièrement dans les revues spécialisées de nos différentes sociétés du monde entier, constitueront toujours à l'avenir la base de la justification des innovations et le garant de notre liberté thérapeutique.

La recherche concomitante et la recherche médicale classique sont deux entités distinctes, mais qui sont complémentaires et doivent être mises en relation dans le cadre de l'introduction de la nouvelle structure tarifaire. Par leurs connaissances, les médecins devront assurer l'interface entre ces divers aspects de la recherche, seule façon pour nos patients d'avoir la garantie d'accès aux traitements appropriés en temps utile.

Dr P.-F. Cuénoud

Membre du Comité Central de la FMH Responsable du domaine SwissDRG 